\title{
EL LIDERAZGO DE SERVICIO Y SU EFECTO TRANSFORMADOR: EL CASO DEL CENTRO DEL MUCHACHO TRABAJADOR
}

SERVANT LEADERSHIP AND ITS TRANSFORMATIONAL EFFECT: THE WORKING BOY'S CENTER CASE

CHRISTIAN BENJAMÍN CABEZAS GUERRA*

Recibido: 6 de agosto de 2016 Aprobado: 27 de septiembre de 2016 



\section{EL LIDERAZGO DE SERVICIO Y SU EFECTO TRANSFORMADOR: EL CASO DEL CENTRO DEL MUCHACHO TRABAJADOR}

Christian Benjamín Cabezas Guerra

PALABRAS CLAVES: Liderazgo, Liderazgo de Servicio, Ecuador, El Centro del Muchacho Trabajador

KEY WORDS: Leadership, Servant-Leadership, Ecuador, The Working Boys' Center

\section{RESUMEN}

En este artículo se presenta el tema del Liderazgo de Servicio (Servant Leadership), la conceptualización que ha recibido este tipo de liderazgo que lo diferencia de otros propuestos en la literatura, sus elementos y las características específicas de los individuos que muestran este liderazgo, los resultados de algunos estudios empíricos sobre la aplicación de este liderazgo en diferentes contextos organizacionales y culturales, y finalmente un estudio de caso realizado sobre este tema en nuestro medio en una institución Jesuita de servicio a los más necesitados. Este tipo de liderazgo, basado en la preocupación primordial de contribuir al desarrollo de los demás, ha sido practicado en el Centro del Muchacho Trabajador (CMT), institución Jesuita de servicio que ha logrado cambiar la vida de decenas de miles de personas en Ecuador. Al final de este trabajo se presentan conclusiones del estudio y sugerencias para futuras investigaciones relacionadas con este tema. 


\section{ABSTRACT}

This article reviews the topic of Servant- Leadership, its conceptualization that makes it different from other types of leadership proposed in literature, its elements and the specific characteristics that Servant Leaders display, the results of various empirical studies developed in different organizational and cultural contexts, and finally, a case study about this topic that was developed in a Jesuit service institution that helps the most needed individuals in our context. This type of leadership, based on the main objective of developing others, has been practiced at The Working Boys' Center and has changed the life conditions of tens of thousands of individuals in Ecuador. The study's conclusions and suggestions for future research related to this topic are presented at the end of this article.

\section{INTRODUCCIÓN}

Cuando se menciona al "liderazgo" como una característica, existe la tendencia de relacionarlo con el manejo del poder y la ocupación de posiciones jerárquicas en diferentes tipos de organizaciones. Aparentemente, esto se ha producido por las conceptualizaciones originales que se le ha dado al mencionado constructo. Remontándonos a las investigaciones iniciales que se dio al liderazgo, Mann (1959) propuso la existencia de una relación positiva entre liderazgo y las características personales de: inteligencia, ajuste, extroversión, dominancia, masculinidad, y sensibilidad. Las primeras teorías de liderazgo propusieron que la principal misión de los líderes era la consecución de objetivos o resultados (McGee-Cooper \& Looper,
2001). Consecuentemente, se asumía que los líderes necesitaban poseer características específicas para influenciar a las personas e inducirlas a trabajar en la consecución de objetivos. Bajo esta estereotipada figura del líder "fuerte" era difícil considerar la posibilidad de que todas las personas pudiesen llegar a ser líderes.

Un aspecto importante que se ha analizado en las últimas décadas sobre el estudio del liderazgo ha sido la pregunta sobre si el liderazgo de las personas es causado por factores innatos o si es producido por influencias situacionales. Sobre este punto tan controvertido, Stogdill (1974) propuso que existen algunas características personales que interactúan con importantes componentes situacionales. Bennis (1989), Gardner 
Revista PUCE, ISSN 1390-7719. Núm.103. 3 de mayo de 2016-3 de NOVIEMBRE DE 2016 ChRISTIAN BENJAMÍn CABEZAS GUERRA. PP，3-31

(1990), y Kotter (1990) han sugerido que el liderazgo puede ser aprendido y desarrollado. Según Gardner (1990, pág. 158), este proceso requiere de una combinación de "motivación, carácter, y oportunidad." Las teorías de Liderazgo Transformacional (Burns, 2003), y de Liderazgo de Servicio (Greenleaf, 1977), han propuesto un rol más humanista del líder que no está únicamente preocupado por alcanzar objetivos, sino que también influencia positivamente en las personas para hacerles crecer. Estas últimas teorías aceptan la posibilidad de que cada individuo pueda ser un agente positivo que ayuda a otros a alcanzar cambios beneficiosos.

De acuerdo con las teorías más recientes, cada individuo podría ser capaz de desempeñarse como un líder, independientemente de su estatus económico o social. Estas teorías rechazan la noción que el liderazgo deba estar asociado únicamente con el uso de posiciones de poder como fuentes de influencia. Estas últimas perspectivas han introducido la noción que el liderazgo se manifieste en servir a otros para apoyarles a crecer y desarrollarse en plenitud como seres humanos. Existen también autores que han sugerido que el liderazgo no es exclusivo de los adultos puesto que puede ser desarrollado y practicado en edades tempranas (Bass \& Stogdill, 1990; Metz, McLellan \& Youniss, 2003). Estos últimos hallazgos apoyan la idea que el liderazgo puede ser aprendido, desarrollado, y practicado por diferentes personas independientemente de su edad, y posición social y económica en nuestras sociedades. No obstante, no queda clara la manera en que se daría este proceso de aprendizaje y desarrollo de liderazgo.

\section{MARCO CONCEPTUAL}

\section{La Evolución de las Teorías del Liderazgo: De Características Personales a Procesos}

El liderazgo es un concepto que históricamente ha recibido mucha atención de investigadores de distintas disciplinas. No obstante, aunque este tema ha sido ampliamente estudiado, su definición y consiguiente operacio- nalización se mantiene ambigua. Burns (1978, pág. 2) afirmó ya hace un buen tiempo que, "El liderazgo es uno de los fenómenos más observados, pero menos entendidos en el planeta". Dado que el liderazgo ha sido estudiado en distintas disciplinas, este fenómeno ha recibido muchas definiciones diferentes. Northouse (2004, pág. 2) afirmó que 
"Existen casi tantas definiciones de liderazgo como personas que han tratado de definirlo." La falta de una definición general aceptada también ha afectado la aplicación del liderazgo para efectos de intervenciones en el desarrollo organizacional. Sobre este tema, Rost (1991) expresó sus dudas sobre la posibilidad de formar líderes cuando no existe una definición consistente, aceptada, y universal de este constructo. Algunos autores han intentado establecer definiciones más generales de liderazgo para reducir la tensión conceptual en torno a este tema. Por ejemplo, Cronin (1980, pág. 392) ofreció una definición simple y concisa de liderazgo como "la capacidad de hacer que las cosas sucedan cuando de otra manera no hubiesen ocurrido". Northouse (2010, pág. 3), en una manera similar, identificó al liderazgo como un proceso en el que un individuo influencia a otros para lograr objetivos comunes.

El liderazgo ha sido comúnmente identificado como un atributo de personas "fuertes" que actúan como los guías de las naciones, organizaciones, grupos, etc. (Carlyle, 1973; Wren, 1995). Han existido diferentes intentos para identificar las características de estos individuos que influencian en otros. El principal interés en identificar estas características está basado en el objetivo de determinar si el liderazgo puede ser enseñado/ aprendido o si tiene componentes innatos. Algunas teorías iniciales defendieron la existencia de características o atributos específicos que son comunes en los líderes en ambientes diferentes. En estas conceptualizaciones iniciales de liderazgo, existió la creencia que existen líderes que nacieron con características diferentes a otras personas que no son consideradas líderes (Bass \& Bass, 2008, pág. 50). Algunos autores han defendido la idea que el liderazgo es el resultado de una interacción entre las personas (que tienen características específicas) y la sociedad (Kelley, 1998; Michelet, 1973; Wren, 1995). De acuerdo con esta idea, existe un componente ambiental que interactúa con las características de los líderes (Northouse, 2015; Smart, 2005). Este argumento es también apoyado por la teoría comportamental que se enfoca en las diferencias situacionales que influencian en el comportamiento de los líderes de acuerdo con su reacción a los contextos (Bass \& Bass, 2008).

En un intento por proporcionar una tipificación de las diferentes teorías de liderazgo, Northouse (2012) propuso la existencia de dos enfoques básicos o categorías amplias para definir al liderazgo como un concepto en la práctica: como características personales y como procesos. El enfoque de características personales defiende la idea de que la gente posee cualidades de liderazgo 
Revista PUCE. ISSN 1390-7719. Núm.103. 3 de Mayo de 2016-3 de NOVIEMBRE DE 2016 Christian BEnJAMÍn CABEZAS GUERRA. PP，3-31

que les predisponen a tener éxito en la ocupación de posiciones de dirección. Una de las primeras teorías basadas en características personales fue la teoría del "Gran hombre" (The Great Man Theory) (Jago, 1982), que defendía que el liderazgo era producido por algunas características físicas como la altura o el atractivo físico. Otros autores sugirieron la existencia de características de liderazgo "fuertes" como algunas habilidades y destrezas, autoconfianza, determinación, persistencia, estatus, fuerza física, ego, etc. (Bass, 1985; Mann, 1959; Stogdill, 1974). Sobre este aspecto, Bass (1985, pág. 45) afirmó, "una característica universal de un líder carismático es su autoconfianza y autoestima". Algunas teorías posteriores incluyeron cualidades sociales como la extroversión y la facilidad en la articulación verbal. Este grupo de teorías han sido criticadas por su naturaleza extremadamente objetiva en tratar un tema tan complejo y su presunción generalizada que si una persona posee esas características, de seguro va a ser un líder natural (Northouse, 1997, pág. 30). Otro criticismo relacionado es la presunción de estas teorías que si alguien no posee las características mencionadas, no podría demostrar liderazgo. Bajo esas presunciones, el liderazgo sería exclusivo de ciertos individuos. Dentro de estas teorías de características personales se encuentran importantes teorías de liderazgo como la del "liderazgo carismático".

De acuerdo con Northouse (2012), aunque las teorías de las características individuales han sido fuertemente criticadas por ser demasiado deterministas y exclusivas, ellas volvieron a ganar popularidad en la segunda mitad del siglo XX. Esto se ha producido principalmente por la afluencia de estudios correlacionales conducidos entre algunas formas de liderazgo como el carismático y el visionario, y características recurrentes demostradas consistentemente por líderes considerados "efectivos" en esos estilos de liderazgo. Debe notarse que algunas teorías recientes de liderazgo, por ejemplo la del Liderazgo Transformacional, de alguna manera reconocen la idea que los líderes demuestran características específicas en su comportamiento. Por ejemplo, han existido estudios relevantes que han logrado identificar características presentes en esas formas de liderazgo (Spears \& Lawrence, 2002).

El enfoque para conceptualizar al liderazgo por procesos defiende la idea que el liderazgo "reside en el contexto de las interacciones entre líderes y seguidores y convierte al liderazgo como algo disponible para todas las personas" (Northouse, 2012, pág. 8). En este enfoque de procesos se reconoce que un aspecto importante del liderazgo reside en la relación que se establece entre líde- 
res y seguidores. Esta perspectiva acepta la posibilidad que el liderazgo sea una característica que puede ser aprendida y perfeccionada por todas las personas. Gardner (1990, pág. 159) sugirió que el liderazgo puede ser aprendido y desarrollado, y que este proceso requiere la combinación de "motivación, carácter, y oportunidad." Este enfoque de procesos no niega que las características personales puedan influenciar la práctica del liderazgo; no obstante también reconoce que existen aspectos situacionales que juegan un rol importante en la práctica del liderazgo. Yukl (1981) sugirió que algunas características pueden incrementar la probabilidad que un individuo pueda ser un líder efectivo, pero la importancia relativa de una característica personal podría depender de la situación. Bass y Stogdill (1990, pág. 59) afirmaron que "ambos elementos, las características individuales y los factores situacionales son importantes, así como la interacción entre ellos". Northouse (2010, pág. 3) notó que "el liderazgo no es linear, un evento de una vía, sino un evento interactivo".

Algunas teorías relevantes de liderazgo como las del liderazgo transaccional, transformacional, y de servicio, ponen énfasis en la importancia de las situaciones y los seguidores en la práctica del liderazgo. Por ejemplo, de acuerdo con Gibb (1969), el liderazgo transaccional propone una interacción recíproca entre un líder y seguidores en la que el líder gana influencia por medio de realizar ajustes críticos, o transacciones con el objetivo de satisfacer las expectativas y necesidades de los seguidores. En la teoría del liderazgo transformacional, el líder "alcanza resultados superiores a través de influenciar en la conciencia de los seguidores sobre los objetivos y los valores" (Bass, 2002, pág. 107).

Burns (1978) diferenció entre el liderazgo transaccional y transformacional sugiriendo que el transaccional seguía un patrón tradicional en el que la relación entre el líder y los seguidores operaba a través de una serie de transacciones o intercambios. Estos intercambios consistían en que el líder entregaba algo al seguidor en intercambio de algo más. Además, en el liderazgo transformacional, los intercambios existen, pero el líder también busca desarrollar la relación a un nivel más elevado en el que la confianza y las expectativas que se establecen con los seguidores son respetadas. Bajo esta forma de liderazgo, Burns (1978) sugiere que un factor de legitimidad envuelve la relación entre el líder y los seguidores y la lleva a otro nivel fundamental. De acuerdo con Northouse (2012), el liderazgo transformacional es un estilo de liderazgo en el que la motivación de los seguidores no es impulsada por una recompensa extrínseca, sino por una motivación intrínseca 
Revista PUCE. ISSN 1390-7719. Núm.103. 3 de mayo de 2016-3 de NOVIEMBRE DE 2016 Christian BEnJAMÍn CABEZAS GUERRA. PP，3-31

y compromiso que está basada en una conexión entre las experiencias entre los seguidores y el líder. Esta conexión transformacional produce una mejora en la moral de ambos, el líder y los seguidores, incitando a que todas las partes constituyentes logren su potencial. Burns (1978, pág. 20) definió al liderazgo transformacional como "algo que ocurre cuando una o más personas se relacionan con otros de tal manera que los líderes y los seguidores levantan unos a otros a niveles más altos de motivación y moral".

La teoría de Liderazgo de Servicio, propuesta por Greenleaf en los años 70, también reconoce la importancia de la relación entre los líderes y los seguido- res. No obstante, esta teoría enfatiza la relevancia de esta relación de una manera única. Greenleaf (1977, pág. 13) afirmó, "La diferencia se manifiesta en el cuidado que el servidor tiene de asegurarse que las necesidades primordiales de los otros estén siendo satisfechas". Greenleaf también sostuvo, "Un gran líder debe ser visto primero como servidor, y ese mismo hecho es la clave de su grandeza" (1977, pág. 21). Este enfoque contrasta con otras formas de liderazgo que se enfocan en el logro de objetivos organizacionales como la prioridad de la práctica del liderazgo, y miran al desarrollo de otros como medios para conseguir esos objetivos (Smith et al., 2004).

\section{EL LIDERAZGO DE SERVICIO: DEFINICIÓN, ELEMENTOS, CARACTERÍSTICAS, Y RESULTADOS DE ESTUDIOS EMPÍRICOS SOBRE SU APLICACIÓN}

"... Un gran líder es primero un servidor..." - Robert Greenleaf (1970).

Robert Greenleaf, mediante un ensayo escrito en 1970, propuso un estilo único de liderazgo basado en enfocarse en las necesidades de los otros. Con su propuesta de que un líder debe ser un servidor primero rompió diferentes paradigmas existentes hasta ese momento en lo que respecta la naturaleza del liderazgo. El Liderazgo de Servicio, con una conceptualización tan simple, pero tan profunda del liderazgo, se ha proliferado en innumerables artículos académicos, libros, material multimedia en las últimas décadas (Smith, 2005).

\section{Definición}

El Liderazgo de Servicio defiende la idea que la primera motivación de un verdadero líder y su rol fundamental es el servicio a los otros. La noción de este 
tipo de liderazgo, también conocido como Liderazgo basado en las necesidades de los demás, tiene una naturaleza paradójica que fue introducida intencionalmente por su proponente. Greenleaf (1970) formuló un concepto único de liderazgo que ha provocado que lectores de sus ensayos se detengan y tomen una pausa para pensar y cuestionar las presunciones históricas sobre el rol de un líder y su relación con los seguidores (Smith, 2005). Greenleaf juntó dos palabras aparentemente contradictorias para proponer que se considere al liderazgo como una característica de una naturaleza diferente a la que a través del tiempo nos ha sugerido la sociedad.

Históricamente, las palabras "servidor" y "servicio" han recibido connotaciones negativas dentro de las pirámides comunes en las diferentes organizaciones. Los servidores usualmente ocupan los niveles más bajos de las jerarquías organizacionales. De acuerdo con Smith (2005, pág. 3), Greenleaf intencionalmente denominó a su primer ensayo sobre el Liderazgo de Servicio como "El servidor como líder" en lugar de "El líder como servidor" para intentar romper en los lectores el modelo mental que establece que un líder podría servir (condición deseable en un líder), cuando el servir debe ser la característica básica y primordial de un líder. De acuerdo con Greenleaf, los servidores naturales, por sus características de desprendimiento, deben ser considerados líderes.

Larry Spears, unos de los principales autores sobre el Liderazgo de Servicio, quien además fue uno de los fundadores y se desempeñó como Director Ejecutivo del "Centro de Liderazgo de Servicio Robert K. Greenleaf" lo definió como:

... Una nueva forma de modelo de liderazgo que coloca el servicio a otros como la prioridad número uno. El liderazgo de Servicio enfatiza elementos importantes como: el servicio a otros, un enfoque holístico del trabajo, la promoción de un sentido de comunidad, y la toma de decisiones compartida (1996, pág. 33).

\section{Elementos del Liderazgo de Servicio}

A continuación se expone una breve explicación de los cuatro principales elementos del Liderazgo de Servicio propuestos inicialmente por Greenleaf:

1. El servicio a los demás. Greenleaf (1996) propuso que el liderazgo de Servicio empieza cuando una persona asume la posición de servicio en las interacciones con los demás para generar crecimiento en ellos. Conforme a esta concepción, el liderazgo es auténtico cuando nace un deseo 
Revista PUCE. ISSN 1390-7719. Núm.103. 3 de mayo de 2016-3 de NOVIEMBRE DE 2016 ChRISTIAN BENJAMÍn CABEZAS GUERRA. PP，3-31

de ayudar a otros y no de intereses individualistas o de ejercicio del poder. Según Greenleaf (1970, pág. 2), el querer ayudar a otros es la"... clave de la grandeza de un líder". Un líder de servicio tiene como primera motivación y objetivo ayudar a otros a desarrollarse y lograr crecer, mientras que, el éxito organizacional es una consecuencia indirecta de ese desarrollo.

\section{Enfoque holístico del trabajo.} Greenleaf (1996, pág. 8) señaló que el Liderazgo de Servicio sostiene que "El trabajo existe para la persona tanto como la persona existe para el trabajo". Esta postura desafía a las organizaciones a repensar las relaciones que se establecen entre las personas a todo nivel organizacional (instituciones y sociedad). Esta teoría promueve que las personas sean estimuladas a ser ellas mismas en su vida personal y profesional. Como afirma Greenleaf, esta valoración más integral de las personas beneficiará a los intereses y rendimiento de las organizaciones.

\section{Promoción de un sentido de comu-}

nidad. En varias de sus obras, Greenleaf expresó su pesar por la pérdida del sentido de comunidad en nuestra sociedad moderna describiéndola como "...el conocimiento gastado de estos tiempos" (1970, pág. 28). El Liderazgo de Servicio cuestiona la habilidad de las instituciones de brindar servicios "humanos" y propone que únicamente la comunidad, definida como grupos de individuos que son co-responsables unos de otros como una entidad, puede desarrollar esa función. Smith (2005) sugirió que solamente estableciendo este sentido de comunidad, las organizaciones pueden llegar a ser exitosas en el cumplimiento de sus objetivos. De acuerdo con Greenleaf (1970, pág. 30), "el sentido de comunidad se puede desarrollar exclusivamente a partir de las acciones de Líderes de Servicio."

\section{Toma de Decisiones Compartida.}

Conforme con esta teoría, un Liderazgo de Servicio efectivo se evidencia en su cultivo en otras personas. El líder de servicio impulsa el desarrollo de una fuerza de trabajo creativa y motivada a través del apoyo a la participación de otros. Russel (2001, pág. 80) describió este proceso como, "Los líderes impulsan a otros a participar, no guardándose el poder que ellos tienen, sino entregándolo completamente". Se ha descrito la estructura organizacional resultante de este tipo de liderazgo como una "pirámide invertida" en la que los empleados, clientes externos, y otros "stakeholders" están en la cima, mientras que los líderes están en la base. 
En un estudio bibliográfico sobre el liderazgo de servicio realizado en años posteriores, Russell (2001) propuso que el liderazgo de servicio, al ser un tipo de liderazgo enfocado en el bienestar de los demás, tiene como elementos influyentes a los valores. Según este autor, "los valores son las variables independientes que impulsan el comportamiento de los líderes de servicio" (Russell, 2001, pág. 82). En ese mismo estudio, Russell identificó como los principales valores del liderazgo de servicio a la humildad, el respeto por los demás, la honestidad, la integridad, la equidad, y el amor. Estos valores específicos identificados por Russell (2001) son congruentes con los elementos propuestos inicialmente por Greenleaf (1996).

\section{Características del Liderazgo de Servicio}

Según Smith, Montagno, y Kuzmenko (2004), usualmente la formulación de teorías y modelos de liderazgo no empieza con un análisis de la motivación del líder. Las teorías generalmente inician con una descripción del liderazgo y las características observables de los líderes. En el caso del Liderazgo de Servicio, por su naturaleza única, la descripción de sus características comienza con un análisis del sistema de creencias y valores del líder, y su motivación para querer liderar. Este tipo de liderazgo involucra principios y valores fuertes como la clave del éxito tanto de los individuos como de las organizaciones. De acuerdo con Patterson (2003a) el Liderazgo de Servicio es una teoría de virtud y valores morales. Esto significa que el líder de servicio, por cuestión de su carácter, buscará hacer lo correcto en cada situación específica.

Algunos investigadores de ciencias del comportamiento han intentado identificar las principales características de este tipo de liderazgo basados en el análisis de los trabajos de Greenleaf. Según a Spears (1996) y Russel y Stone (2002), las 10 principales características de este tipo de liderazgo son las siguientes:

1. Escucha activa. De acuerdo con Greenleaf, "Solamente un verdadero líder de servicio responde automáticamente a cualquier problema mediante escuchar primero" (1970, pág. 10).

2. Empatía. Según Greenleaf, "El servidor siempre acepta y da importancia a otros, nunca rechaza" (1970, pág. 12). El líder de servicio procura entender a la persona en su situación existencial sin emitir juicios anticipados.

3. Sanación. Un líder de servicio reconoce el deseo humano de desarrollarse y de ayudar a otros a que se desarrollen. Greenleaf definió "sanar" 
Revista PUCE, ISSN 1390-7719. Núm.103. 3 de mayo de 2016-3 de NOVIEMBRE DE 2016 ChRISTIAN BENJAMÍn CABEZAS GUERRA. PP，3-31

como "Hacer completo al otro" (1970, pág. 27).

4. Atención. Un líder de servicio mantiene una permanente atención en los diferentes elementos que interactúan en el contexto. De acuerdo con Greenleaf, sin atención, "perdemos oportunidades de liderazgo" (1970, pág. 19).

5. Persuasión. Greenleaf (1970) enfatizó la importancia de la persuasión "amable" pero "persistente" para lograr objetivos de crecimiento en otros. La persuasión no debe usarse para ejercer poder o lograr objetivos personales. Greenleaf sugirió que en la actualidad ya se analizan de una manera más fresca y positiva los temas del poder y la autoridad, y la gente está comenzando a aprender a relacionarse de una manera menos coercitiva y más de apoyo (1970).

6. Conceptualización. Según Greenleaf (1970), el líder de servicio puede abstraer y crear soluciones a problemas que todavía no se presentan porque puede analizar y conceptualizar los contextos.

7. Previsión. Greenleaf expuso que la "Previsión sobre lo que va a suceder en el futuro es mejor que una adivinación al azar" (1970, pág. 16). Esta característica es relevante en un líder de servicio para enfrentar dificultades de la mejor manera posible.
8. Administración responsable. LOS administradores de las instituciones no solamente deben preocuparse de lo mejor para personas específicas, sino para la institución como un todo y su impacto y relación con la sociedad (Greenleaf, 1970).

\section{Compromiso con el crecimiento de} la gente. Consiste en una valoración del bienestar de los otros. Para Greenleaf, "El secreto del engrandecimiento de una institución consiste en conformar un equipo de personas que se hagan crecer los unos a los otros más de lo que podrían si no formasen parte del equipo" (1970, pág. 14).

10. Generación de comunidad. De acuerdo con Greenleaf (1970), el establecimiento de nuestras grandes instituciones modernas que compiten entre sí ha afectado la unidad de las personas en la sociedad. Nuestra sociedad se ha convertido en extremadamente individualista. Greenleaf afirmó que "Lo que es necesario para reconstruir a la sociedad es que existan suficientes líderes de servicio que nos muestren el camino" (1970, pág. 30).

\section{Diferencia entre el Liderazgo de Servicio y Otros Estilos Contemporáneos de Liderazgo}

Como se mencionó previamente, el Liderazgo de Servicio nació como una nueva propuesta algo diferente a 
otros estilos de liderazgo que aparecieron en la literatura en las últimas décadas. En estudios sobre el Liderazgo de Servicio se ha analizado principalmente sus diferencias con el liderazgo transformacional. De acuerdo con Stone, Russell, y Patterson (2004), la diferencia principal entre el liderazgo de servicio y el transformacional es que el primero se enfoca claramente en los colaboradores mientras que el segundo en los objetivos organizacionales. Patterson (2003b) propuso que a diferencia del liderazgo transformacional propuesto por Burns (2003), el Liderazgo de Servicio tiene la ventaja de poseer constructos claros inherentes al mismo. En un estudio desarrollado en el año 2003, Patterson propuso un modelo conceptual en el que identificó 7 constructos como fundamentos del liderazgo de servicio: "amor moral del líder, humildad, altruismo, visión, confianza, empoderamiento, y servicio" (2003b, pág. 7).

En un estudio empírico realizado por Parolini (2007) se investigó la diferenciación entre los componentes del liderazgo de servicio y el transformacional a través de encuestas. El análisis de ítems reflejó que las principales diferencias de estos dos estilos de liderazgo consistían en 5 áreas claves: moral, enfoque, motivo, misión, desarrollo e influencia. En estas 5 áreas, el Liderazgo de Servicio se diferenció en que la preocupación primordial del mismo se centra en el desarrollo y bienestar de las personas mientras que en el transformacional se enfoca en el desarrollo organizacional y consecución de objetivos. En un análisis posterior sobre estos resultados iniciales, Parolini, Patterson, y Winston (2009, pág. 288) concluyeron que estas diferencias "deben ser integradas en prácticas de liderazgo y desarrollo organizacional para saber diferenciar entre la necesidad de contar con un líder transformador o de servicio...". Estos mismos autores reconocieron que los dos estilos de liderazgo muestran ventajas y desventajas y pueden ser aplicados según las necesidades diferentes en las organizaciones.

Es relevante mencionar que aunque varios estudios han señalado diferencias especificas entre el liderazgo de servicio y el transformacional, esto no resulta evidente para los colaboradores de las instituciones. Algunos trabajadores perciben ambos estilos de liderazgo como muy parecidos. En un estudio correlacional realizado en 5 empresas públicas y privadas de Estados Unidos, Washington, Sutton, y Sauser (2014), midieron la percepción de los empleados en cuanto a la diferencia de varios estilos de liderazgo mostrados por sus supervisores. Este estudio encontró que el Liderazgo de Servicio se percibía fuerte y positivamente relacionado con el liderazgo transformacional, y negativamen- 
Revista PUCE. ISSN 1390-7719. Núm.103. 3 de mayo de 2016-3 de NOVIEMBRE DE 2016 ChRISTIAN BENJAMÍn CABEZAS GUERRA. PP，3-31

te relacionado con el liderazgo pasivo y el liderazgo "laissez faire".

\section{Críticas al Liderazgo de Servicio}

Aunque el Liderazgo de Servicio ha ganado una creciente popularidad en las últimas décadas, también ha recibido críticas por parte de algunos autores. Por ejemplo, Russell (2001, pág. 145) destacó las bondades de esta propuesta de liderazgo, no obstante también admitió que la misma es "... sistemáticamente indefinida y no suficientemente apoyada por investigación empírica". Como una crítica adicional Eicher-Catt (2005) ha sugerido que a pesar que esta forma de liderazgo promueve aparentemente la práctica de valores, la misma contiene un discurso retórico contradictorio (servir y liderar) que acentúa sesgos de género e inequidades puesto que nuestras sociedades tienen roles muy fuertemente establecidos. De acuerdo con esta autora, esta teoría defiende un mito irrealizable puesto que en nuestras sociedades no sería posible mostrar la orientación de servir y al mismo tiempo liderar.

\section{Estudios Empíricos Sobre la Aplicación del Liderazgo de Servicio}

A pesar de las críticas mencionadas, algunos estudios empíricos más recientes han mostrado varias eviden- cias de los beneficios de adoptar el Liderazgo de Servicio dentro de diversos tipos de organizaciones. Por ejemplo, en una extensa revisión de la bibliografía existente sobre el Liderazgo de Servicio, Van Dierendonck (2010) presentó un resumen de diferentes hallazgos empíricos que demuestran los beneficios de este estilo de liderazgo. De acuerdo con Van Dierendonck (2010, pág. 21) existe abundante evidencia empírica de estudios transversales que demuestra que los líderes de servicio "hacen que los empleados estén más satisfechos, comprometidos, y muestren mejor desempeño" en comparación con otros tipos de líderes. Según este mismo autor, los meta-análisis sobre el liderazgo de servicio demuestran que existen 3 cambios específicos en los empleados que perciben este tipo de liderazgo en sus organizaciones: crecimiento personal en términos de autorealización, cambio en lo que respecta a mejora de actitudes hacia el trabajo y transformación en términos de trabajo en equipo y colaboración con otros.

En otro estudio desarrollado con el objetivo de evaluar el verdadero impacto del Liderazgo de Servicio en los contextos organizacionales, Parris y Peachey (2013) utilizaron la metodología de revisión sistemática de literatura (RSL) para analizar en conjunto los resultados de 39 investigaciones en con- 
textos organizacionales en los que se aplicó este tipo de liderazgo. Los resultados de este amplio análisis mostraron que la teoría de liderazgo de servicio es viable y ayuda a mejorar el bienestar de los empleados en estos contextos. Una de las principales conclusiones de este estudio fue que "la teoría del Liderazgo de Servicio es aplicable en una variedad de culturas, contextos, y ambientes organizacionales" (Parris \& Peachey, 2013, pág. 388).

También se han realizado estudios sobre la aplicación de este tipo de liderazgo en distintos contextos culturales. Por ejemplo, Mahembe y Engelbrecht (2014) en una investigación realizada en instituciones educativas en Sudáfrica, encontraron una relación positiva fuerte entre el liderazgo de servicio, efectividad de equipos de trabajo, y comportamientos organizacionales positivos. Estos autores concluyeron que la aplicación de este tipo de liderazgo resulta conveniente en instituciones en las cuales se establecen objetivos claros de desarrollo de las personas, empoderamiento y reconocimiento de diferentes talentos.

Otros estudios han analizado la aplicabilidad de este estilo del liderazgo considerando diferencias en componentes culturales nacionales. Como se mencionó anteriormente, los valores son una base fundamental que influen- cia los estilos de liderazgo, en especial al Liderazgo de Servicio. Diferentes estudios han demostrado que los valores culturales nacionales no son estáticos ni compartidos en todo el mundo (Hofstede, 2001). De acuerdo con Rubio-Sanchez, Bosco, y Melchar (2013), la diferencia de valores culturales entre países produciría que el estilo de Liderazgo de Servicio pueda tener mejor aceptación y resultados en culturas en las cuales se apoyan valores similares a los que este estilo de liderazgo promulga. Estos mismos autores examinaron la congruencia de los valores que promueve este tipo de liderazgo con los valores nacionales de diferentes países identificados en la encuesta mundial de valores (World Values Survey). Los resultados de esta investigación mostraron que este tipo de liderazgo podría encajar mejor en algunos países que en otros. De acuerdo con Rubio-Sánchez, Bosco, y Melchar (2013), el Liderazgo de Servicio sería de mejor aplicabilidad en Estados Unidos, Bangladesh, Suecia, Canadá, y Tanzania, mientras que sería de menor aplicabilidad en Rusia, Lituania, Letonia, Bielorrusia, Ucrania, Estonia, y Turquía. Los autores concluyeron que este tipo de liderazgo encajaría de mejor manera en culturas en las cuales se valora la iniciativa, libertad de decisión, confianza, y autonomía. 
Revista PUCE, ISSN 1390-7719. Núm.103. 3 de mayo de 2016-3 de

\section{EL LIDERAZGO DE SERVICIO: EL CASO DEL CENTRO DEL MUCHACHO TRABAJADOR (CMT)}

\section{Antecedentes}

Aunque el concepto de Liderazgo de Servicio fue introducido por Greenleaf hace más de cuatro décadas, y el mismo ha sido tema de números estudios en varios países, en especial en Estados Unidos y Europa, este concepto no ha recibido notable atención en América Latina en donde son muy escasos los estudios realizados sobre este tipo importante de liderazgo (Irving \& Mcintosh, 2009). En esta sección se presenta un estudio realizado con el propósito de identificar los factores que influenciaron a los niños que ingresaron en la obra de inspiración ignaciana del Centro del Muchacho Trabajador (CMT) a que rompan junto a sus familias el ciclo de pobreza en el cual estaban sumergidos. En este estudio también se exploró al liderazgo de servicio como generador del cambio positivo que lograron los beneficiarios de esta institución.

Una figura reconocida de liderazgo en el CMT ha sido el Padre John Halligan, S.J., fundador y cabeza principal de esta institución desde su creación en el año 1964. Este destacado líder del CMT recibió en el año 2010 el Premio Opus (Opus Award), galardón que a nivel mundial se otorga a católicos que han logrado con su trabajo y sacrificio cambios considerables y soluciones a problemas de índole global. Desde su creación el CMT ha logrado cambiar la condición de pobreza de más de 30.000 personas en nuestro país (Calle, 2007). De acuerdo con el Padre Halligan (comunicación personal, 2014), el logro de este gran objetivo se ha producido principalmente porque se ha provisto a los niños y a sus familias de programas educacionales y otras actividades que están direccionadas hacia la formación, desarrollo, y refuerzo de valores positivos que consecuentemente producen cambios en las actitudes y comportamientos de esos individuos. Los valores que se forjan en el CMT se enfocan principalmente en direccionar a los individuos a ser ciudadanos responsables que apoyen el crecimiento de otros. De esa manera se logra el crecimiento de la comunidad de manera recíproca.

En un estudio previo conducido por Calle (2007), que midió el impacto del centro en las vidas de los niños y sus familias que participaron de esta iniciativa, se encontró que más del $75 \%$ de esos individuos mejoraron sus condiciones como resultado de las experiencias que tuvieron en el centro. La mayoría de esas personas pudieron avanzar en sus estudios luego de graduarse en el CMT, 
empezaron sus propios negocios y adquirieron sus propias viviendas. El mencionado estudio logró medir y evidenciar el fuerte impacto que esa institución ha tenido en la sociedad ecuatoriana. Sin embargo, quedó pendiente investigar los mecanismos por los cuales se logró ese cambio positivo en los participantes del CMT. Cabe señalar que la gran mayoría de estudios sobre niños trabajadores de la calle que se han realizado a nivel global se han limitado a realizar una descripción de sus comportamientos, problemas y características de los programas de ayuda. Existen muy pocos estudios a nivel mundial que han tratado los mecanismos de cambios de comportamiento en estos individuos.

\section{Objetivos}

El presente estudio de caso tuvo dos objetivos principales: el primero fue identificar los factores que afectaron positivamente a los beneficiarios del CMT a lograr un cambio positivo. El segundo objetivo fue investigar si el estilo de liderazgo del Padre John Halligan, S.J, es congruente con las características del Liderazgo de Servicio.

\section{Participantes}

En este estudio participaron una muestra de 175 beneficiarios del CMT, 5 trabajadores, 5 voluntarios y las 2 principales autoridades del Centro. Los par- ticipantes no fueron sujetos a muestreo probabilístico. No obstante, se realizó una estratificación de ellos de acuerdo con las fases de la historia de la institución.

\section{Métodos}

Para cumplir con el primer objetivo de determinar los factores que influenciaron en la notable transformación que tuvieron los participantes del CMT se utilizaron métodos mixtos en dos fases. En la primera fase se utilizaron métodos cualitativos (entrevistas y grupos focales) con la muestra de beneficiarios, trabajadores, voluntarios y autoridades del CMT para posteriormente, a través de análisis de contenido, lograr identificar de una manera general los factores que influenciaron el cambio. En la segunda fase, únicamente los beneficiarios aplicaron el método del ordenamiento de tarjetas (pile-sort method) para establecer un "ranking" de relevancia de los principales elementos identificados en la primera fase. En esta segunda fase del estudio, para poder identificar diferencias estadísticamente significativas entre los elementos ordenados por los beneficiarios, se utilizó de acuerdo con lo sugerido por Nussbaum (2014) para estos casos, la prueba de Friedman. Posteriormente, para analizar diferencias específicas entre elementos, se realizaron posthoc análisis aplicando el test Wilcoxon con una corrección de Bonferroni. 
Revista PUCE. ISSN 1390-7719. Núm.103. 3 de mayo de 2016-3 de NOVIEMBRE DE 2016 ChRISTIAN BENJAMÍn CABEZAS GUERRA. PP，3-31

Para el cumplimiento del segundo objetivo de este estudio, la misma muestra de participantes proporcionó información sobre las características de liderazgo manifestadas por el Padre Halligan en sus interacciones con ellos. Esta información también fue sujeta a análisis de contenido. En este análisis se identificaron las características que según los participantes fueron más relevantes en su proceso de cambio y que además fueron mencionadas con mayor frecuencia por ellos en las entrevistas y grupos focales. Se utilizó el número de menciones de los participantes como criterio para identificar los elementos más salientes de acuerdo con lo sugerido por Esterberg (2002) para este tipo de análisis. Posteriormente se procedió a evaluar si las características identificadas eran congruentes con las sugeridas en la literatura sobre el Liderazgo de Servicio.

\section{Resultados}

\section{Factores que impulsaron el cambio en los beneficiarios del CMT}

Los resultados del análisis de la información obtenida con el método de ordenamiento de tarjetas, mostró en primer lugar que el principal factor que influenció en el cambio de los participantes del CMT fue el "cambio de valores" a través de las diferentes actividades lideradas por el Padre Halligan y la Hermana Conway, principales autoridades del Centro. Este elemento tuvo diferencias estadísticamente significativas en comparación con todos los otros elementos identificados (valores $\mathrm{p}<0.001$ ).

Algunos otros elementos considerados como de gran importancia por los beneficiarios para lograr su cambio positivo fueron: haber incluido a su familia en el programa (característica relevante y diferente del CMT), haber aprendido a valorar a través del Centro a los otros miembros de sus familias, haber aprendido el valor del trabajo, haber aprendido espiritualidad, haber aprendido a valorar la responsabilidad, haber recibido cuidado y amor, y haber aprendido a ser autosuficientes. Cabe destacar que los elementos identificados como los de menor importancia por los participantes fueron los servicios y beneficios "gratuitos" que brinda el centro como son la alimentación gratuita (13 en el "ranking") y los servicios de salud gratuitos (14 en el "ranking"). Estos dos últimos elementos también presentaron una diferencia estadísticamente significativa comparados con todos los otros considerados como "más importantes" por los beneficiarios (valor $p<0.002$ ). Los resultados de este primer análisis cuantitativo se muestran en la tabla 1. 
Tabla 1: Resultados de la prueba Wilcoxon con una corrección de Bonferroni aplicados a los principales elementos del CMT.

\begin{tabular}{|c|c|c|c|c|c|c|c|c|c|c|c|c|c|c|}
\hline $\begin{array}{c}\text { Elementos del CMT ordenados por } \\
\text { los particpantes según su } \\
\text { relevancia }\end{array}$ & \begin{tabular}{|c|} 
Aprender \\
valores
\end{tabular} & 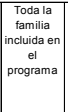 & \begin{tabular}{|c|} 
El CMT \\
influenció a \\
los \\
participantes \\
a valorar a \\
sus propias \\
familias
\end{tabular} & \begin{tabular}{|c} 
El CMT \\
influenció a \\
los \\
participantes \\
a valorar su \\
propio \\
trabajo
\end{tabular} & \begin{tabular}{|c|} 
EI CMT \\
enseñó a los \\
participantes \\
espiritualidad
\end{tabular} & \begin{tabular}{|c|} 
El CMT \\
influenció a \\
los \\
participantes \\
a valorar la \\
responsabili \\
dad
\end{tabular} & \begin{tabular}{|c|} 
El CMT \\
brindó a los \\
participantes \\
amory \\
cuidado
\end{tabular} & $\begin{array}{c}\text { Aprender a } \\
\text { ser auto- } \\
\text { suficientes }\end{array}$ & \begin{tabular}{|c|} 
Aprender \\
una carrera \\
técnica
\end{tabular} & \begin{tabular}{|c|} 
El CMT \\
influenció a \\
los \\
participantes \\
a valorara el \\
ahorro
\end{tabular} & $\begin{array}{c}\text { El CMT les } \\
\text { brindó } \\
\text { acceso a la } \\
\text { educación }\end{array}$ & \begin{tabular}{|c|} 
Aprender \\
colaboración \\
\end{tabular} & $\begin{array}{c}\text { Alimentación } \\
\text { gratuita }\end{array}$ & \begin{tabular}{|c|}
$\begin{array}{c}\text { Servicios de } \\
\text { salud } \\
\text { gratuitos }\end{array}$ \\
\end{tabular} \\
\hline Aprender valores & & $0.000^{* *}$ & $0.000^{* *}$ & $0.000^{* *}$ & $0.000^{* *}$ & $0.000^{*+*}$ & $0.000^{* *}$ & $0.000^{* *}$ & $0.000^{* *}$ & $0.000^{*+*}$ & $0.000^{* *}$ & $0.000^{* *}$ & $0.000^{* *}$ & $0.000^{* *}$ \\
\hline $\begin{array}{l}\text { Toda la familia incluida en el } \\
\text { programa }\end{array}$ & $0.000^{* *}$ & & 0,645 & 0,108 & 0,088 & 0,075 & 0,031 & 0,010 & $0.003^{*}$ & $0.003^{*}$ & $0.002^{*}$ & $0.002^{\star}$ & $0.000^{* *}$ & $0.000^{* *}$ \\
\hline $\begin{array}{l}\text { EI CMT influenció a los participantes } \\
\text { a valorar a sus propias familias }\end{array}$ & $0.000^{* *}$ & 0,645 & & 0,290 & 0,055 & 0,100 & 0,023 & 0,008 & 0,004 & $0.003^{*}$ & 0,004 & $0.002^{\star}$ & $0.000^{* *}$ & $0.000^{* *}$ \\
\hline $\begin{array}{l}\text { El CMT influenció a los participantes } \\
\text { a valorar su propio trabajo }\end{array}$ & $0.000^{+*}$ & 0,108 & 0,290 & & 0,518 & 0,471 & 0,268 & 0,081 & 0,028 & 0,031 & 0,054 & 0,011 & $0.000^{* *}$ & $0.000^{* *}$ \\
\hline \begin{tabular}{|l|} 
EI CMT enseñó a los participantes \\
espiritualidad
\end{tabular} & $0.000^{* *}$ & 0,088 & 0,055 & 0,518 & & 0,852 & 0,316 & 0,084 & 0,094 & 0,100 & 0,092 & 0,036 & $0.000^{+*}$ & $0.000^{* *}$ \\
\hline $\begin{array}{l}\text { EI CMT influenció a los participantes } \\
\text { a valorar la responsabilidad }\end{array}$ & $0.000^{* *}$ & 0,075 & 0,100 & 0,471 & 0,852 & & 0,311 & 0,120 & 0,092 & 0,080 & 0,054 & 0,079 & $0.000^{* *}$ & $0.000^{* *}$ \\
\hline $\begin{array}{l}\text { El CMT brindo a los participantes } \\
\text { amor y cuidado }\end{array}$ & $0.000^{* \star}$ & 0,031 & 0,023 & 0,268 & 0,316 & 0,311 & & 0,341 & 0,320 & 0,372 & 0,344 & 0,256 & $0.000^{* *}$ & $0.000^{* *}$ \\
\hline Aprender a ser autosuficientes & $0.000^{+*}$ & 0,010 & 0,008 & 0,081 & 0,084 & 0,120 & 0,341 & & 0,604 & 0,559 & 0,709 & 0,400 & $0.000^{* *}$ & $0.000^{* *}$ \\
\hline Aprender una carrera técnica & $0.000^{+*}$ & $0.003^{*}$ & 0,004 & 0,028 & 0,094 & 0,092 & 0,320 & 0,604 & & 0,585 & 0,791 & 0,581 & $0.000^{* *}$ & $0.000^{* *}$ \\
\hline $\begin{array}{l}\text { EI CMT influenció a los participantes } \\
\text { a valorar el ahorro }\end{array}$ & $0.000^{* *}$ & $0.003^{*}$ & $0.003^{*}$ & 0,031 & 0,100 & 0,080 & 0,372 & 0,559 & 0,585 & & 0,940 & 0,775 & $0.001^{\star}$ & $0.000^{\star *}$ \\
\hline $\begin{array}{l}\text { EI CMT les brindó acceso a la } \\
\text { educación }\end{array}$ & $0.000^{* *}$ & $0.002^{\star}$ & 0,004 & 0,054 & 0,092 & 0,054 & 0,344 & 0,709 & 0,791 & 0,940 & & 0,999 & $0.000^{* *}$ & $0.000^{* *}$ \\
\hline Aprender colaboración & $0.000^{* *}$ & $0.002^{\star}$ & $0.002^{\star}$ & 0,011 & 0,036 & 0,079 & 0,256 & 0,400 & 0,581 & 0,775 & 0,999 & & $0.000^{* *}$ & $0.000^{* *}$ \\
\hline Alimentación gratuita & $0.000^{+*}$ & $0.000^{* *}$ & $0.000^{* *}$ & $0.000^{* *}$ & $0.000^{* *}$ & $0.000^{* *}$ & $0.000^{* *}$ & $0.000^{* *}$ & $0.000^{+*}$ & $0.001^{\star}$ & $0.000^{* *}$ & $0.000^{* *}$ & & 0,124 \\
\hline Servicios de salud gratuitos & $0.000^{+*}$ & $0.000^{* \star}$ & $0.000^{* *}$ & $0.000^{* *}$ & $0.000^{* \star}$ & $0.000^{* *}$ & $0.000^{* *}$ & $0.000^{\star *}$ & $0.000^{* *}$ & $0.000^{\star \star}$ & $0.000^{* *}$ & $0.000^{* *}$ & 0,124 & \\
\hline
\end{tabular}

${ }^{*} p<.004$

${ }^{* *} p<.001$

\section{Características de Liderazgo de Servicio del Padre Halligan}

Como un segundo objetivo del estudio, los participantes del CMT identificaron las características del liderazgo del Padre Halligan que fueron relevantes en la modificación de sus valores. Las principales características de liderazgo del Padre Halligan identificadas por los participantes de este estudio, así como el número de menciones de estas cualidades por los participantes identificadas como producto del análisis de contenido se presentan en la tabla 2. 
Revista PUCE. ISSN 1390-7719. Núm.103. 3 de mayo de 2016-3 de NOVIEMBRE DE 2016 Christian BEnJAMÍn CABEZAS GUERRA. PP，3-31

Tabla 2: Características de liderazgo manifestadas por el Padre Halligan en el CMT

\begin{tabular}{|c|c|}
\hline $\begin{array}{l}\text { Características de liderazgo demostradas por el Padre Halligan } \\
\text { en sus interacciones en el CMT }\end{array}$ & $\begin{array}{c}\text { Número de } \\
\text { menciones de } \\
\text { participantes }\end{array}$ \\
\hline $\begin{array}{l}\text { El Padre Halligan influenció al Centro y a sus participantes con su servicio y } \\
\text { trabajo fuerte para ayudar a los más necesitados. }\end{array}$ & 37 \\
\hline El Padre Halligan motivó a los participantes a colaborar unos con otros. & 36 \\
\hline El Padre Halligan influenció al Centro y a sus participantes con su humildad. & 32 \\
\hline $\begin{array}{l}\text { El Padre Halligan influenció al Centro y a sus participantes con su } \\
\text { espiritualidad. }\end{array}$ & 23 \\
\hline $\begin{array}{l}\text { El Padre Halligan influenció al Centro y a sus participantes con amor, } \\
\text { amabilidad y empatía. }\end{array}$ & 22 \\
\hline $\begin{array}{l}\text { El Padre Halligan enseñó diferentes valores a los participantes con la práctica } \\
\text { de los mismos. }\end{array}$ & 22 \\
\hline El Padre Halligan influenció a los participantes con su guía personal. & 22 \\
\hline $\begin{array}{l}\text { El Padre Halligan enseñó a los participantes a valorarse más y a ser más } \\
\text { autosuficientes (sanación). }\end{array}$ & 20 \\
\hline $\begin{array}{l}\text { El Padre Halligan influenció al Centro y a sus participantes con su } \\
\text { perseverancia. }\end{array}$ & 17 \\
\hline $\begin{array}{l}\text { El Padre Halligan influenció al Centro y a sus participantes con su liderazgo } \\
\text { basado en su preocupación por los demás. }\end{array}$ & 17 \\
\hline $\begin{array}{l}\text { El Padre Halligan influenció al Centro y a sus participantes con su liderazgo } \\
\text { basado en su solidaridad. }\end{array}$ & 14 \\
\hline
\end{tabular}

Al analizar las características presentes en el Padre Halligan identificadas por los beneficiarios, fue posible notar que algunas de ellas corresponden claramente a características del Liderazgo de Servicio propuestas en la literatura (Spears, 1996; Russel y Stone, 2002). Las características propuestas por Spears (1996) pueden agruparse en tres diferentes segmentos: características personales comunicacionales como la escucha activa, empatía, sanación, y persuasión; características cognitivas como la atención, conceptualización, y previsión; y características de orientación a la comunidad como la administración responsable, compromiso con el crecimiento de la gente y generación de comunidad.

De la información obtenida en esta fase de la investigación se pudo concluir que el Padre Halligan evidenció claramente la práctica de las características de liderazgo de servicio comunicacionales y de orientación a la comunidad. Por ejemplo, en lo que se refiere a las características comunicacionales, de acuerdo con la mayoría de los beneficiarios, el Padre Halligan demostró en sus 
interacciones con ellos características como empatía, sanación y persuasión para producir un cambio positivo. Muchos de ellos consideraron al Padre Halligan como un guía que no los juzgaba. El escuchaba y los ayudaba cuando afrontaban dificultades. También persuadía a los beneficiarios a enfocarse en objetivos positivos y a colaborar con otros. Estos comportamientos son consistentes con lo que Greenleaf describió como un rasgo común de un líder de servicio. Greenleaf (1977, pág. 33) propuso: "El líder de servicio acepta a los otros y les da importancia, nunca los rechaza".

En lo que respecta a las características de orientación a la comunidad, el Padre Halligan evidenció algunas de las propuestas por Spears (1996) como por ejemplo: administración responsable, compromiso con el crecimiento de los otros, y generación de comunidad. De acuerdo con el testimonio de los beneficiarios, trabajadores, y voluntarios del CMT, cuando el Centro ha enfrentado dificultades económicas en las más de 5 décadas de existencia, el Padre Halligan siempre ha encontrado soluciones a las crisis. Las soluciones se han basado principalmente en una administración responsable de los recursos en tiempos de crisis económica, creación de nuevos emprendimientos en conjunto con los beneficiarios y trabajo en consecución de fondos internacionales. El Padre Halligan también se ha enfocado en el crecimiento de las personas proporcionándoles herramientas para poder mejorar. Los participantes fueron capaces de mejorar su calidad de vida no solamente con una educación formal, sino también, y principalmente con la formación en valores. Ellos fueron enseñados a colaborar y servir. De acuerdo con el Padre Halligan (comunicación personal, 2015), ellos se convirtieron en generadores de cambio para otros que afrontaron dificultades similares. El tipo de liderazgo basado en "servir primero" demostrado por el Padre Halligan claramente encaja con lo que Greenleaf (1996) describió como la característica central de un líder de servicio. Esta característica importante, manifestada por el Padre Halligan, influenció a los participantes del CMT a convertirse también en líderes de servicio.

\section{DISCUSIÓN DE RESULTADOS Y CONCLUSIONES}

En este artículo se ha presentado una revisión bibliográfica de la teoría del liderazgo de servicio propuesta por
Greenleaf (1977) en el contexto de las diferentes teorías de liderazgo formuladas a través del tiempo. Las características de 
Revista PUCE. ISSN 1390-7719. Núm.103. 3 de Mayo de 2016-3 de NOVIEMBRE DE 2016 Christian BEnJAMÍn CABEZAS GUERRA. PP，3-31

este tipo de liderazgo resultan novedosas en los contextos organizacionales comunes en los que se asumen a los líderes como individuos "dominantes y fuertes" que son seguidos por otros más "débiles". El liderazgo de servicio, aunque no haya sido difundido de una manera amplia en nuestro medio, ya ha sido practicado de una manera efectiva por individuos que han llevado la misión de apoyar y ayudar a otros a mejorar sus condiciones de vida, y al mismo tiempo les han influenciado a que sean autosuficientes y contribuyan al cambio positivo de otros.

Las características de liderazgo del principal líder del Centro del Muchacho Trabajador, identificadas por los participantes del estudio, nos brindan evidencia que el modelo de liderazgo de servicio propuesto por Greenleaf (1977) está presente en manifestaciones espontáneas. Los hallazgos en el CMT nos hacen considerar que es posible que el tipo de liderazgo basado en las necesidades de los otros tenga resultados positivos, no necesariamente en un rédito económico para organizaciones específicas, sino en un rédito social de calidad de vida para nuestras naciones.

Un hallazgo importante del estudio en el CMT ha sido que el liderazgo de servicio manifestado por el Padre Halligan en sus interacciones con los beneficiarios ha sido aprendido por estos últimos. Los beneficiarios manifestaron en las entrevistas y grupos focales que la influencia positiva del Centro, y en particular del Padre Halligan, produjo un cambio en su comportamiento y les condujo a convertirse en personas más solidarias y entregadas al bienestar de los demás. Esto fue corroborado por los voluntarios y personal administrativo del Centro. En el CMT se originan permanentemente diferentes emprendimientos orientados hacia el bienestar común. Muchos de esos emprendimientos nacen como iniciativas de los mismos beneficiarios. Este hallazgo también proporciona evidencia que sustenta la propuesta teórica de Greenleaf (1977) en lo que respecta a la posibilidad que este tipo de liderazgo pueda desarrollarse en otros. Es decir, es un liderazgo que al parecer no está determinado por factores innatos sino que se aprende mediante influencias positivas. Este hallazgo también es congruente con lo encontrado en la revisión de Van Dierendonck (2010) sobre resultados de estudios empíricos de Liderazgo de Servicio. De acuerdo con este autor, uno de los principales resultados de la práctica de este tipo de liderazgo en contextos organizacionales es el desarrollo del mismo estilo por parte de los demás miembros de la organización.

Cabe mencionar que históricamente lo niños trabajadores de la calle han sido rechazados por la sociedad por diferentes estereotipos que han recibi- 
do. A ellos se les han asignado atributos negativos que han reforzado la creencia errónea que no podrían ser líderes que influencien positivamente a otros (Thomas de Benítez, 2011). En el CMT se evidenció que los beneficiarios pudieron cambiar su condición de pobreza y al mismo tiempo contribuir a cambiar esa misma condición en otros. Los hallazgos en el CMT han demostrado la posibilidad de formación de liderazgo en menores de edad propuesta por Metz, McLellan, y Youniss (2003) y Sacks (2009). También se demostró que personas que enfrentan grandes privaciones como los niños trabajadores de la calle pueden convertirse en líderes. Estos hallazgos ayudan a crear una nueva orientación a replantear en nuestro contexto el carácter humanista del liderazgo.

Sobre ese rol de liderazgo que han manifestado los beneficiarios del CMT, el Padre Halligan (comunicación personal, 2015) describió:

Si alguien quiere aprender algo significativo sobre el liderazgo, debe venir al CMT y entrevistar a nuestros líderes. El Centro en la actualidad es manejado por personas que fueron niños trabajadores de la calle. Ellos conducen el Centro. Ellos forman equipos de líderes que trabajan con la gente y organizan maneras para progresar, ayudar, y mantenerse saludables.

Una de las características más salientes del liderazgo de servicio propuesto por Greenleaf (1977) es la motivación inicial de servicio de este tipo de líderes. Greenleaf puso mucho énfasis en que un líder de servicio debe inicialmente escoger el servicio antes que liderar. Esa motivación inicial lo convierte en un verdadero líder. De acuerdo con Greenleaf (1977, pág. 27), la mejor manera de identificar este tipo de liderazgo en una persona es preguntarse si: "Aquellos a los que les brinda servicio crecen como personas? Se convierten en individuos más saludables, libres, autónomos, y es más probable que se conviertan también en servidores?" Los hallazgos del estudio en el CMT demuestran que el Padre Halligan tuvo esa influencia de transformación descrita por Greenleaf en los beneficiarios del Centro.

Como se expresó previamente, una de las principales maneras para explicar y clasificar las diferentes teorías de liderazgo existentes es dividirlas de acuerdo con su énfasis en características personales o en procesos (Northouse, 2012). Los resultados de la investigación en el CMT son consistentes con el enfoque de procesos que pone mucho énfasis en el efecto que tienen las interacciones entre los líderes y seguidores 
Revista PUCE. ISSN 1390-7719. Núm.103. 3 de mayo de 2016-3 de NOVIEMBRE DE 2016 Christian BEnJAMÍn CABEZAS GUERRA. PP，3-31

en el desarrollo de liderazgo en estos últimos. El Centro ha provisto del contexto para diferentes interacciones que han producido un importante crecimiento en los participantes, lo que les ha conducido a demostrar liderazgo de servicio. En este análisis es importante mencionar la posibilidad de aprender y desarrollar liderazgo propuesta por Gardner (1990, pág. 158) para lo cual se requiere de: motivación, carácter, y oportunidad. Los diferentes desafíos que los beneficiarios del CMT enfrentaron en su proceso de cambio significaron una prueba fuerte para medir su motivación y carácter.

\section{RECOMENDACIONES PARA FUTURAS INVESTIGACIONES SOBRE EL TEMA}

Este estudio de caso se ha enfocado en un segmento de individuos que han sufrido algunos tipos de carencias significativas y que han logrado cambiar su condición de vida a través de la influencia de un programa liderado por un individuo que mostró características congruentes con las propuestas en la teoría del Liderazgo de Servicio. El CMT ofrece un programa integral que presenta diferentes elementos que han tenido un efecto de transformación en sus participantes. Como esta investigación se ha enfocado en un tipo de liderazgo específico por parte de una persona que ha liderado un programa único por sus características en un contexto determinado, sería muy interesante comparar los elementos detectados en este estudio con otros que están presentes en programas similares en otros contextos. Al identificar los elementos comunes que han producido un cambio positivo en diferentes contextos se podrían proponer políticas públicas de apoyo a individuos en condiciones similares de carencias.

En este estudio se ha evidenciado que los niños trabajadores de la calle podrían convertirse en líderes positivos que influencian en otros que sufren dificultades similares. Con el contexto propicio y oportunidades, ellos han demostrado que pueden convertirse en líderes de servicio que ayudan a otros a crecer. Este hallazgo apoya la noción que las personas, independientemente de su condición económica o social, pueden ser capaces de practicar liderazgo si se les brinda la oportunidad y las condiciones necesarias. No obstante, dentro de la información obtenida en este estudio se pudo conocer que unos pocos participantes dejaron el Centro sin haber alcanzado ese resultado deseado. 
Sería importante investigar los elementos que causaron esa deserción en esos participantes y que les impidió lograr esa transformación.

\section{Bibliografía}

Bass, B. M. (1985). Leadership and performance beyond expectations. New York: The Free Press.

Bass, B. M. (2002). Cognitive, social, and emotional intelligence of transformational leaders. In Riggio, R. E., Murphy, S. E., \& Pirozzolo, F. J. (Eds.), Multiple Intelligences and leadership. Mahwah, NJ: Lawrence Erlbaum Associates, Inc.

Bass, B. M., \& Stogdill, R. M. (1990). Bass \& Stogdill's handbook on leadership (3rd ed.). New York: The Free Press.

Bass, B. M., \& Bass, R. (2008). The Bass Handbook of Leadership: Theory, Research and Managerial Application. New York, NY: Free Press.

Bennis, W. (1989). On becoming a leader. Wilmington, MA: Addison-Wesley.

Burns, J. M. (2003). Transforming leadership: a new pursuit of happiness. New York: Grove Press.

Burns, J. M. (1978). Leadership. New York: Harper and Row.

Calle, M. A. (2007). 42 años abriendo caminos de dignidad-evaluación externa de impacto de la obra del Centro del Muchacho Trabajador Una Familia de Familias. Quito: Crissan Color.

Carlyle, T. (1973). History as Biography: Thomas Carlyle on history. In F. Stern (Ed.), The varieties of history: From Voltaire to the present (pp. 90-107). New York: Vintage Books. 
Revista PUCE. ISSN 1390-7719. Núm.103. 3 de mayo de 2016-3 de NOVIEMBRE DE 2016 Christian BEnJAMÍn CABEZAS GUERRA. PP，3-31

Cronin, T. (1980). The state of the presidency (2nd ed.). Boston: Little, Brown.

Eicher-Catt, D. (2005). The myth of servant-leadership: A feminist perspective. Women and Language, 28(1), 17-26. Recuperado de http://proquest.umi.com/ pqdweb/?did $=859822181 \& \mathrm{Fmt}=7 \&-$ clientld $=46781 \& R Q T=309 \& V$ Name $=P Q D$

Esterberg, Kristin G. (2002). Qualitative methods in social research. Boston: McGraw-Hill.

Gardner, J. W. (1990). On leadership. New York: The Free Press.

Gibb, C.A. (1969). Leadership. Middlesex, UK: Putnam.

Greenleaf, R. K. (1970/2002). Servant leadership: A journey into the nature of legitimate power and greatness. New York: Paulist Press.

Greenleaf, R.K. (1996). On becoming a servant-leader. San Francisco: Josey-Bass Publishers.

Gregory Stone, A., Russell, R.F. and Patterson, K. (2004) 'Transformational versus servant leadership: A difference in leader focus, Leadership \& Organization Development Journal, 25(4), pp. 349-361.

Hofstede G (2001). Cultural consequences: Comparing values, behaviors, institutions, and organizations across nations, (2nd ed). Thousand Oaks, CA: Sage.

Irving, J., \& Mcintosh, T. (2009). Investigating the Value of and Hindrances to Servant Leadership in the Latin American Context: Initial Findings from Peruvian Leaders. Journal of International Business and Cultural Studies, 2, 1-16.

Jago, A. G. (1982). Leadership: Perspectives in theory and research. Management Science, 28(3), 315-336.

Kelley, R. E. (1998). Followership in a leadership world. In L. C. Spears (Ed.), Insights on Leadership: Service, stewardship, and servant leadership (pp. 170-184). New York: John Wiley \& Sons.

Kotter, J. P. (1990). A force for change: How leadership differs from management. New York: Free Press.

Mahembe, B., \& Engelbrecht, A. S. (2014). The relationship between servant leadership, organisational citizenship behaviour and team effectiveness. SA Journal of Industrial Psychology, 40(1).

Mann, R. D. (1959). A review of the relationships between personality and performance in small groups. Psychological Bulletin, 56, 241-270.

Metz, E., McLellan, J., \& Youniss, J. (2003). Types of Voluntary Service and Adolescents' Civic Development. Journal of Adolescent Research, 18(2), 188-203.

McGee-Cooper, A., \& Looper, G. (2001). The essentials of ServantLeadership: Principles in Practice. Waltham, MA: Pegasus.

Michelet, J. (1973). History as a national epic: Michelet. In Stern, F. (Ed.), 
The varieties of history: From Voltaire to the present (pp. 108-119). New York: Vintage Books.

Northouse, P. (1997). Leadership theory and practice. Thousand Oaks, CA: Sage Publications.

Northouse, P. (2004). Leadership theory and practice. Thousand Oaks, CA: Sage Publications.

Northouse, P. (2010). Leadership theory and practice. Thousand Oaks, CA: Sage Publications.

Northouse, P. (2012). Leadership theory and practice. Thousand Oaks, CA: Sage Publications.

Northouse, P. (2015). Leadership theory and practice. Thousand Oaks, CA: Sage Publications.

Parolini, J. L. (2007). Investigating the distinctions between transformational and servant leadership. ProQuest Dissertations and Theses, (February), 103-n/a. Recuperado de $\quad$ http://search.proquest.com/ docview/304712710?accountid=34574

Nussbaum, M. (2014). Categorical and Nonparametric Data Analysis: Choosing the Best Statistical Technique. New York, NY: Routledge.

Parolini, J., Patterson, K., \& Winston, B. (2009). Distinguishing between transformational and servant leadership. Leadership \& Organization Development Journal, 30(3), 274-291.
Parris, D. L., \& Peachey, J. W. (2013). A Systematic Literature Review of Servant Leadership Theory in Organizational Contexts. Journal of Business Ethics, 113(3), 377-393.

Patterson, K.A. (2003a). Servant Leadership: A Theoretical Model. Tesis Doctoral, Regent University.

Patterson, K. A. (2003b). Servant leadership: A theoretical model. Regent University School of Leadership Studies: Servant Leadership Research Roundtable - August 2003, (March), 1-10.

Rost, J. C. (1991). Leadership for the twenty-first century. Westport, CT: Praeger.

Rubio-Sánchez, A., Bosco, S. M., \& Melchar, D. E. (2013). Servant Leadership and World Values. Global Studies Journal, 5(3), 19-33.

Russell, R.F. (2001). The role of values in servant leadership. Leadership \& Organization Development Journal, 22(2), 76-83.

Russell, R.F., and Stone, A.G. (2002). A review of servant leadership attributes: developing a practical model. Leadership \& Organization Development Journal, 23(3), 145-157.

Sacks, R. E. (2009). Natural born leaders: An exploration of leadership development in children and adolescents (Order No. NR61078). Available from ProQuest Dissertations and Theses A\&l: 
Revista PUCE. ISSN 1390-7719. Núm.103. 3 de mayo de 2016-3 de NOVIEMBRE DE 2016 Christian Benjamín CABEZAS GUERRA. PP，3-31

The Humanities and Social Sciences Collection. (577366584). Recuperado de http://search.proquest.com/docview/577366584?accountid $=1557$

Smart, M. (2005). The role of informal leaders in organizations: The hidden organizational asset. Tésis doctoral inédita, University of Idaho.

Smith, B. N., Montagno, R. V., \& Kuzmenko, T. N. (2004). Transformational and servant leadership: Content and contextual comparisons. Journal of Leadership \& Organizational Studies (Baker College), 10(4), 80-91. Recuperado de http://search.ebscohost.com/login. asp $x$ ? direct $=r$ true $\& d b=b t h \& A N=T$ 474649 I\&site =ehost-live

Smith, C. (2005). Servant leadership: the leadership theory of Robert K. Greenleaf. Leadership Quarterly, (6), 1-17.

Spears, L. (1996). Reflections on Robert K. Greenleaf and servant-leadership. Leadership \& Organization Development Journal, 17(7), 33-35.

Spears, L., and Lawrence, M. (Eds.). (2002). Focus on leadership: ServantLeadership for the 21st century. New York: John Wiley \& Sons.

Stogdill, R. M. (1974). Handbook of leadership. New York: The Free Press.

Thomas de Benitez, S. (2011). State of the World's Street Children: Research, Street Children Series, Street Children Consortium, London, UK.
Van Dierendonck, D. (2010). Servant Leadership: A Review and Synthesis. Journal of Management, 37(4), 1228-1261. $\quad$ http://doi. org/10.1177/0149206310380462

Washington, R. R., Sutton, C. D., \& Sauser, J. W. I. (2014). How distinct is servant leadership theory? Empirical comparisons with competing Theories. Journal of Leadership, Accountability \& Ethics, 11(1), 11-25.

Wren, J.T. (Ed.). (1995). The leader's companion: Insights on leadership through the ages. New York: The Free Press.

Yukl, G. A. (1981). Leadership in organizations. Englewood Cliffs, NJ: Prentice Hall. 
